\title{
Clinical Significance of Lymph Node Micrometastasis in pNo Gastric Cancer Patients
}

\author{
Yu Li $(\mathbb{D}$, Dongsheng Wang, Yi Li, Xiaodong Liu, Dong Chen, Chentong Yuan, \\ and Yanbing Zhou \\ Department of Gastrointestinal Surgery, The Affiliated Hospital of Qingdao University, Qingdao, Shandong, China \\ Correspondence should be addressed to Yu Li; liyu11920@hotmail.com and Yanbing Zhou; zhouyanbing11205@126.com
}

Received 2 May 2020; Revised 11 February 2021; Accepted 23 February 2021; Published 3 March 2021

Academic Editor: Roberto Caronna

Copyright (c) $2021 \mathrm{Yu} \mathrm{Li}$ et al. This is an open access article distributed under the Creative Commons Attribution License, which permits unrestricted use, distribution, and reproduction in any medium, provided the original work is properly cited.

\begin{abstract}
Purpose. To investigate the relationship between lymph node micrometastasis (LNMM) and clinicopathological factors and to evaluate the prognostic effects of LNMM in pN0 gastric cancer (GC) patients. Methods. One hundred and seventy-two GC patients who received radical gastrectomy with D2 lymph node dissection were enrolled in the present study. 1371 negative lymph nodes from level 2 station confirmed by pathology were examined. The LNMM was diagnosed by telomeric repeat amplification protocol/enzyme-linked immunosorbent assay (TRAP-ELISA). The relationship between clinicopathological factors and LNMM was investigated by multivariate analysis. Survival analysis was performed to evaluate the effects of LNMM on prognosis. Results. LNMM was detected in 423 lymph nodes from 72 patients. The results showed that invasion depth $(\mathrm{OR}=3.755, P=0.004)$, TNM staging $(\mathrm{OR}=3.152, P=0.002)$, lymphatic invasion $(\mathrm{OR}=2.178, P=0.009)$, and tumor differentiation $(\mathrm{OR}=1.266, P=0.013)$ were independent risk factors associated with LNMM. Survival analysis showed that patients with LNMM had significantly worse 5 -year survival compared with those without LNMM $(42 \%$ vs. $76.4 \%, P<0.05)$. Multivariate analysis demonstrated that LNMM, tumor size, Lauren type, invasion depth, and lymphatic invasion $(P<0.05)$ were independently factors associated with 5 -year survival. Conclusions. The findings showed that tumor invasion depth, TNM staging, lymphatic invasion, and tumor differentiation were independent risk factors associated with LNMM occurrence. Moreover, LNMM is a clinically negative prognostic factor in pN0 GC patients.
\end{abstract}

\section{Introduction}

Gastric cancer (GC) is the fifth most frequently diagnosed cancer and the third leading cause of cancer death worldwide, which is responsible for over 1,000,000 new cases and an estimated 783,000 deaths in 2018 [1]. Despite the progress in surgical techniques and oncologic therapies, the prognosis of GC patients is disappointing, with a 5-year overall survival rate remaining at $28 \%$ in most areas of the world, except in Japan, where a 5-year survival rate is up to 70\% [2]. Lymph node metastasis is currently considered to be one of the most significant prognostic factors in GC patients. Therefore, radical gastrectomy with $\mathrm{D} 2$ lymph node dissection is recognized as the standard surgical treatment of GC in East Asia [3].

However, despite curative resection of primary tumor and lymphadenectomy, some patients with histologically node-negative GC still die of local or distant tumor recur- rence [4-6]. Previous studies suggest that lymph node micrometastasis (LNMM) may be one of the key causative factor for GC recurrence [7]. According to the 8th edition of American Joint Committee on Cancer (AJCC) staging system, micrometastasis (MM) is defined as tumor deposits greater than $0.2 \mathrm{~mm}$ but less than $2.0 \mathrm{~mm}$ in largest dimension that is negative by conventional histological examinations, but positive by advanced diagnostic tools [8]. Although prior studies have attempted to clarify the relationship between LNMM and clinicopathological features and to explore the prognostic value of LNMM in GC patients $[5,9,10]$, the clinical significance of MM remains controversial currently.

With the rapid development of molecular biology technology, such as immunohistochemistry (IHC) and reverse transcription-polymerase chain reaction (RT-PCR), the detection rate of MM has increased significantly [11]. Telomerase is a specialized cellular reverse transcriptase that uses 


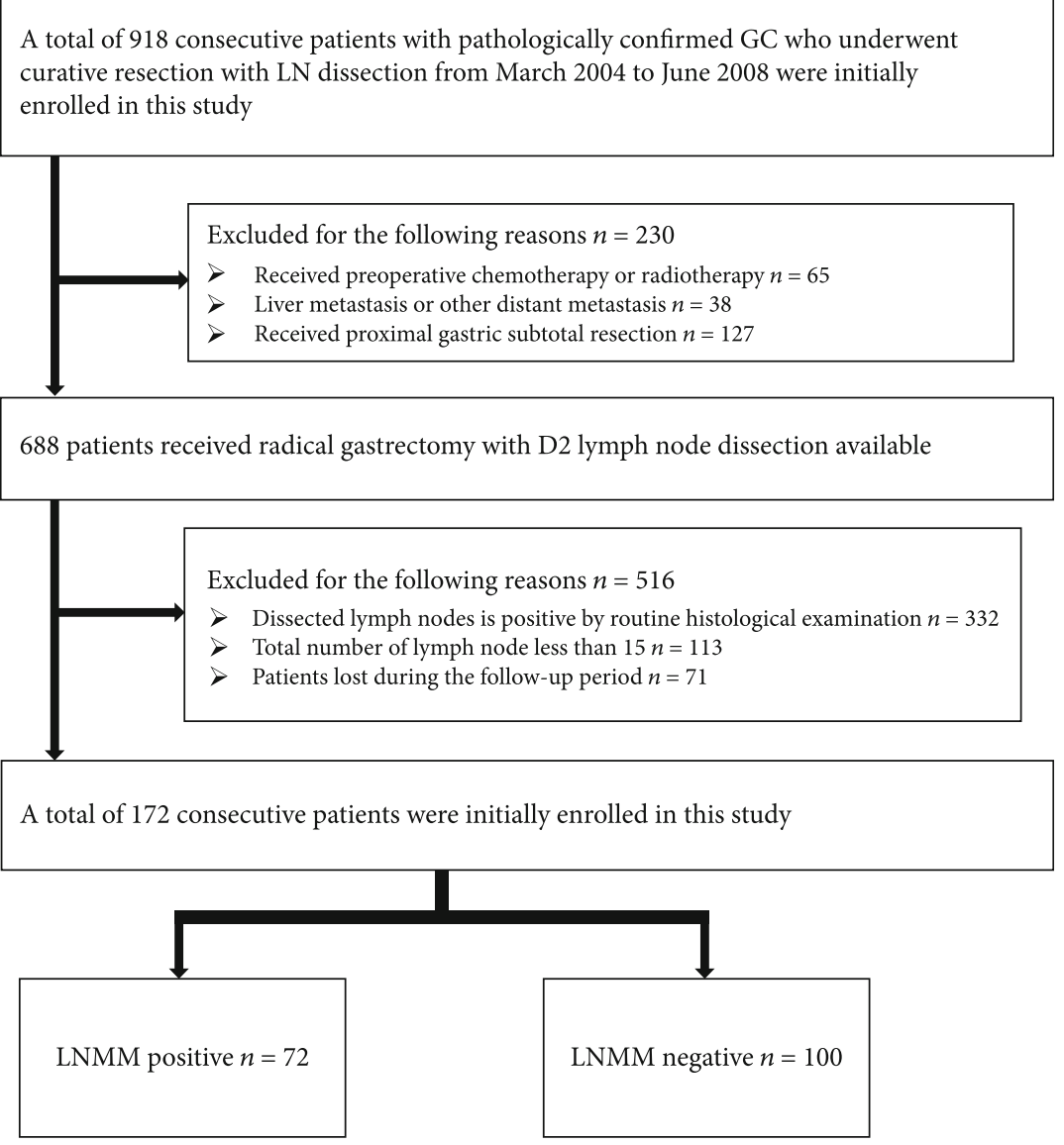

FIGURE 1: Recruitment pathway for patients in this study.

its RNA (Ribonucleic acid) template to elongate the telomere by adding G-rich telomeric repeats to its terminal $3{ }^{\prime}$ overhang and is strongly suppressed in human somatic cells; however, robust telomerase activity is seen in highly proliferative tissues as well as in cancer cells [12]. Several studies have indicated that telomerase reactivation plays an important role in gastric carcinogenesis, and telomerase activity can be used as a marker for neoplastic transformation in GC [13]. Hu et al. reported that telomeric repeat amplification protocol/enzyme-linked immunosorbent assay (TRAPELISA) was significantly more sensitive than cytology or CA125 assay in detecting early peritoneal metastasis [14]. However, to the best of our knowledge, few studies have used this technique to detect LNMM in GC patients.

Therefore, the aim of this study is to investigate the incidence of LNMM in pN0 GC patients using TRAP-ELISA, to explore the relationship between LNMM and clinicopathological factors and to evaluate the prognostic value of LNMM in 5-year survival.

\section{Materials and Methods}

2.1. Patients. Ethical approval was obtained from the medical ethics committee of the Affiliated Hospital of Qingdao University for this retrospective study. 918 consecutive patients diagnosed of GC who received gastrectomy with D2 lymph node dissection from March 2004 to June 2008 in the Affili- ated Hospital of Qingdao University were chosen for this study. All the patients were admitted according to the patient recruitment pathway using the inclusion and exclusion criteria described in Figure 1. A total of 172 patients were incorporated into our study finally.

Clinical data, such as age, gender, and surgical approach, were collected by reviewing medical records. Tumor size, depth of invasion, tumor differentiation, Lauren classification, lymphatic infiltration, and neural invasion were obtained from pathological reports directly. TNM staging was established postoperatively by surgical oncologists and pathologists according to the American Joint Committee on Cancer (AJCC) TNM staging system, the 8th edition [8].

2.2. Specimens Collection. The dissected lymph nodes were classified according to the Japanese Classification of Gastric Carcinoma, $3^{\text {rd }}$ English edition [15], and the No. 7, 8a, 9, $11 \mathrm{p}, 12 \mathrm{a}$ lymph nodes were defined as the level 2 station lymph nodes. Macroscopic lymph nodes from GC specimens were obtained within 15 to 30 minutes after dissection during the operation. Each fresh lymph node specimen was divided equally into two halves. One half was prepared for routine histological examinations, and the other half was immediately frozen in liquid nitrogen for TRAP-ELISA.

2.3. TRAP-ELISA. Frozen samples were mixed with precooled cell lysis reagent, homogenized and incubated on ice 
for $30 \mathrm{~min}$, and then centrifuged at $16000 \mathrm{r} / \mathrm{min}$. The supernatant was collected for the TRAP assay using TRAP reaction kit (Medac, Wedel, Germany). For each sample to be tested, a $50 \mu \mathrm{l}$ reaction mixture containing taq DNA polymerase, primers (CS5' CCCTTACCCTTACCCTTACCCCA $3^{\prime}$; TS $5^{\prime}$ AATCCGTCGTCG AGCCAGAGTT $3^{\prime}$ ), and $3 \mu \mathrm{g}$ protein extraction from the supernatant was prepared. Each reaction mixture was subjected to primer extension at $25^{\circ} \mathrm{C}$ for $20 \mathrm{~min}$, telomerase inactivation at $94^{\circ} \mathrm{C}$ for $5 \mathrm{~min}$ followed with incubation at $90^{\circ} \mathrm{C}$ for $3 \mathrm{~min}$. Then, each reaction mixture was subjected to $35 \mathrm{PCR}$ cycles of denaturation at $50^{\circ} \mathrm{C}$ for 30 seconds and extension at $72^{\circ} \mathrm{C}$ for $90 \mathrm{~s}$, followed by a final cycle of extension at $72^{\circ} \mathrm{C}$ for $90 \mathrm{~s}$. Finally, the reaction mixtures were incubated at $4^{\circ} \mathrm{C}$ for reaction termination.

ELISA was performed as described previously [14]. Briefly, $5 \mu \mathrm{l}$ amplification product was mixed with $20 \mu \mathrm{l}$ denaturation reagent and incubated at room temperature for $10 \mathrm{~min}$. Then, $255 \mu \mathrm{l}$ of hybridization buffer was added into the mixture. $100 \mu \mathrm{l} /$ well of the mixture was added into a precoated microtiter plate (MTP) module and incubated at $37^{\circ} \mathrm{C}$ on a shaker $(300 \mathrm{rpm})$ for $2 \mathrm{~h}$. After adding $100 \mu \mathrm{l} /$ well anti-digoxigeninperoxidase (DIG-POD) working solution, the MTP module was incubated at room temperature for $30 \mathrm{~min}$ while shaking at $300 \mathrm{rpm}$. The reaction mixture was then removed, and $100 \mu \mathrm{l}$ anti-DIG-POD working solution was added per well and incubated at room temperature for $30 \mathrm{~min}$ while shaking at $300 \mathrm{rpm}$. The wells were then washed 5 times with a washing buffer for a minimum of $30 \mathrm{~s}$ each. After the final wash, $100 \mu \mathrm{l} /$ well tetramethylbenzidine substrate solution was added and incubated at room temperature for 10-20 min while shaking at $300 \mathrm{rpm}$. The absorbance value for each sample was calculated as Absorbance (units) $=\mathrm{A} 450-\mathrm{A} 690$. Lysis supernatant inactivated at $85^{\circ} \mathrm{C}$ for $10 \mathrm{~min}$ was used as a negative control. As positive control, we used an extract of telomerase positive embryonic kidney cell line 293, provided with the kit. Negative controls were prepared from positive controls, considering that telomerase essentially requires integrity of its internal RNA component as a template for the addition of the telomeric repeat sequences to the telomerase-specific primer [16].

2.4. Follow-Up and Statistical Analysis. Patients were followed up for disease recurrence and long-term survival until the end of the study (Dec 25, 2014) or disease recurrence or death. Association between patients' clinical characteristics and LNMM was analyzed by multivariate analysis using the Cox proportional hazards model. Variables were analyzed using the $\chi^{2}$ test or Fisher's exact test. The Kaplan-Meier method was used to estimate overall survival. Prognostic factors for 5-year survival were analyzed using Cox proportional hazards regression model. $P<0.05$ was considered statistically significant. Statistical analysis was performed using SPSS version 18.0 for Windows (SPSS, Chicago, Illinois, USA).

\section{Results}

3.1. Patients. From March 2004 to June 2008, a total of 172 patients with node-negative GC were enrolled in our study.
Among the 172 patients, the mean age was $57.36 \pm 12.13$ years. All of the patients received radical gastrectomy (119 patients with distal gastrectomy and 53 patients with total gastrectomy) and D2 lymphadenectomy. A total of 4125 negative lymph nodes were obtained from 172 patients, among which 1371 lymph nodes were from level 2 station, and the average number of resected lymph nodes was $23.84 \pm 9.04$. Based on the TRAP-ELISA analysis, 423 level 2 station lymph nodes from 72 patients $(41.86 \% 72 / 172)$ had LNMM, including 8 lymph nodes from two early gastric cancer patients.

3.2. Clinicopathological Factors Associated with LNMM. Associations between clinicopathological characteristics and LNMM are shown in Table 1. The following clinicopathological variables were significantly associated with LNMM: tumor size, tumor differentiation, the depth of tumor invasion, lymphatic infiltration, Lauren classification, and TNM staging. As the depth of tumor extends, the rate of LNMM also increases. LNMM was found in 10.53\% (2/19), 30.77\% $(8 / 26), 45.54 \%(46 / 101)$, and $61.54 \%(16 / 26)$ of patients with T1 (mucosa and submucosa), T2 (muscularis propria), T3 (subserosa), and T4 (serosal layer or beyond) depth invasion, respectively. Multivariate analysis showed that tumor differentiation ( $\mathrm{OR}=1.266,95 \% \mathrm{CI} 4.274-37.037, P=0.013)$, lymphatic infiltration $(\mathrm{OR}=2.178,95 \%$ CI 1.635-6.327, $P=0.009)$, the depth of tumor invasion ( $\mathrm{OR}=3.755,95 \%$ CI 1.716-8.218, $P=0.004)$, and TNM staging $(\mathrm{OR}=3.152$, 95\% CI 1.547-7.589, $P=0.002)$ were independent risk factors associated with LNMM (Table 2).

3.3. Overall Survival. Patients were followed up for long-term survival (Figure 2). The median follow-up time was 37.5 (range 1.2-137.0) months. Fourteen patients were lost during follow-up, and the follow-up rate was $91.9 \%$. Seventy-five patients died due to recurrent gastric carcinoma. The 1-, 3-, 5 -year overall survival rates were $75 \%, 64 \%$, and $56 \%$, respectively. Five-year overall survival (OS) rate was significantly longer in patients without LNMM vs. with LNMM $(76.4 \%$ vs. $42 \%, P<0.001)$.

Univariate analysis demonstrated that tumor size $(P=0.01)$, tumor differentiation $(P<0.01)$, the depth of tumor invasion $(P=0.01)$, lymphatic infiltration, Lauren classification $(P=0.01)$, TNM staging $(P=0.01)$, and LNMM $(P=0.01)$ were significantly associated with 5 -year overall survival (Table 3). Additional multivariate analysis showed that level 2 station LNMM $(\mathrm{HR}=2.476,95 \% \mathrm{CI}$ $1.415-4.334, \quad P=0.001)$, the depth of tumor invasion $(\mathrm{HR}=1.418,95 \%$ CI 1.060-1.897, $P=0.019)$, lymphatic infiltration $(\mathrm{HR}=2.893,95 \% \mathrm{CI} 1.697-4.934, P=0.00)$, Lauren classification $(\mathrm{HR}=0.209,95 \% \mathrm{CI} 1.083-1.527, P=0.001$ ), tumor size $(\mathrm{HR}=1.978,95 \% \mathrm{CI} 1.260-3.106, P=0.003)$, and TNM staging $(\mathrm{HR}=1.375,95 \% \mathrm{CI} 1.024-1.846, P=$ 0.034 ) were independent risk factors of 5-year survival (Table 4). Further analysis showed that LNMM was an independent prognostic indicator for patients with histologically node-negative T1-T3 GC. However, in patients with T4 disease, the prognostic value of LNMM was not significant $(P=0.568)$. 
TABLE 1: Clinicopathological factors of the patients associated with LNMM.

\begin{tabular}{|c|c|c|c|}
\hline \multirow{2}{*}{$\begin{array}{l}\text { Clinicopathological } \\
\text { factors, } n(\%)\end{array}$} & \multicolumn{2}{|c|}{ LNMM } & \multirow[t]{2}{*}{$P$ value } \\
\hline & Yes & No & \\
\hline Gender & & & 0.059 \\
\hline Male & $56(77.8)$ & $66(66.0)$ & \\
\hline Female & $16(22.2)$ & $34(34.0)$ & \\
\hline Age & & & 0.749 \\
\hline$\leq 60$ & $40(55.6)$ & $58(58.0$ & \\
\hline$\geq 60$ & $32(44.4)$ & $42(42.0)$ & \\
\hline Tumor size $(\mathrm{cm})$ & & & $0.031^{*}$ \\
\hline$\leq 2$ & $6(8.3)$ & $25(25.0)$ & \\
\hline $2 \sim 5$ & $37(51.4)$ & $42(42.0)$ & \\
\hline$\geq 5$ & $29(40.3)$ & $33(33.0)$ & \\
\hline Tumor differentiation & & & $0.032^{*}$ \\
\hline $\begin{array}{l}\text { High or moderate } \\
\text { differentiation }\end{array}$ & $13(18.1)$ & $34(34.0)$ & \\
\hline Low differentiation & $59(81.9)$ & $66(66.0)$ & \\
\hline The depth of tumor invasion & & & $0.001^{*}$ \\
\hline $\mathrm{T} 1$ & $2(2.8)$ & $17(17.0)$ & \\
\hline $\mathrm{T} 2$ & $8(11.1)$ & $18(18.0)$ & \\
\hline $\mathrm{T} 3$ & $46(63.9)$ & $55(55.0)$ & \\
\hline $\mathrm{T} 4$ & $16(22.2)$ & $10(10.0)$ & \\
\hline Surgical approach & & & 0.636 \\
\hline Total gastrectomy & $49(68.1)$ & $70(70.0)$ & \\
\hline Distal gastrectomy & $23(31.9)$ & $30(30.0)$ & \\
\hline General type & & & 0.181 \\
\hline Topical type & $23(31.9)$ & $42(42.0)$ & \\
\hline Infiltrative type & $49(68.1)$ & $58(58.0)$ & \\
\hline Lauren classification & & & $0.034^{*}$ \\
\hline Diffusive type & $51(70.8)$ & $53(53.0)$ & \\
\hline Intestinal type & $21(29.2)$ & $47(47.0)$ & \\
\hline TNM staging & & & $0.005^{*}$ \\
\hline IA & $2(2.8)$ & $17(17.0)$ & \\
\hline IB & $8(11.1)$ & $18(18.0)$ & \\
\hline IIA & $46(63.9)$ & $55(55.0)$ & \\
\hline IIB & $11(15.3)$ & $8(8.0)$ & \\
\hline III & $5(6.9)$ & $2(2.0)$ & \\
\hline Lymphatic infiltration & & & $0.043^{*}$ \\
\hline Yes & $47(65.3)$ & $54(54.0)$ & \\
\hline No. & $25(34.7)$ & $46(46.0)$ & \\
\hline Neural invasion & & & 0.067 \\
\hline Yes & $36(50.0)$ & $46(46.0)$ & \\
\hline No & $36(50.0)$ & $54(54.0)$ & \\
\hline
\end{tabular}

$n$ : number; LNMM: lymph node micrometastasis; TNM: tumor node metastasis; $P$ value was derived from the univariable association analyses between each characteristic; ${ }^{*} P<0.05$.

\section{Discussion}

In the present study, we found a higher LNMM rate (72/172, $41.86 \%)$ in pN0 GC patients. The multivariate logistic analy-
TABLE 2: Multivariate analysis of clinicopathological factors associated with LNMM

\begin{tabular}{lcc}
\hline Variables & OR $(95 \%$ CI $)$ & $P$ value \\
\hline Tumor differentiation & $1.266(4.274-37.037)$ & $0.013^{*}$ \\
Lymphatic infiltration & $2.178(1.635-6.327)$ & $0.009^{*}$ \\
The depth of tumor invasion & $3.755(1.716-8.218)$ & $0.004^{*}$ \\
Lauren classification & $1.256(0.513-3.631)$ & 0.157 \\
Tumor size & $1.907(0.706-5.156)$ & 0.203 \\
TNM staging & $3.152(1.547-7.589)$ & $0.002^{*}$ \\
\hline
\end{tabular}

LNMM: lymph node micrometastasis; TNM: tumor node metastasis; OR: odd ratio; CI: confidence interval; ${ }^{*} P<0.05$.

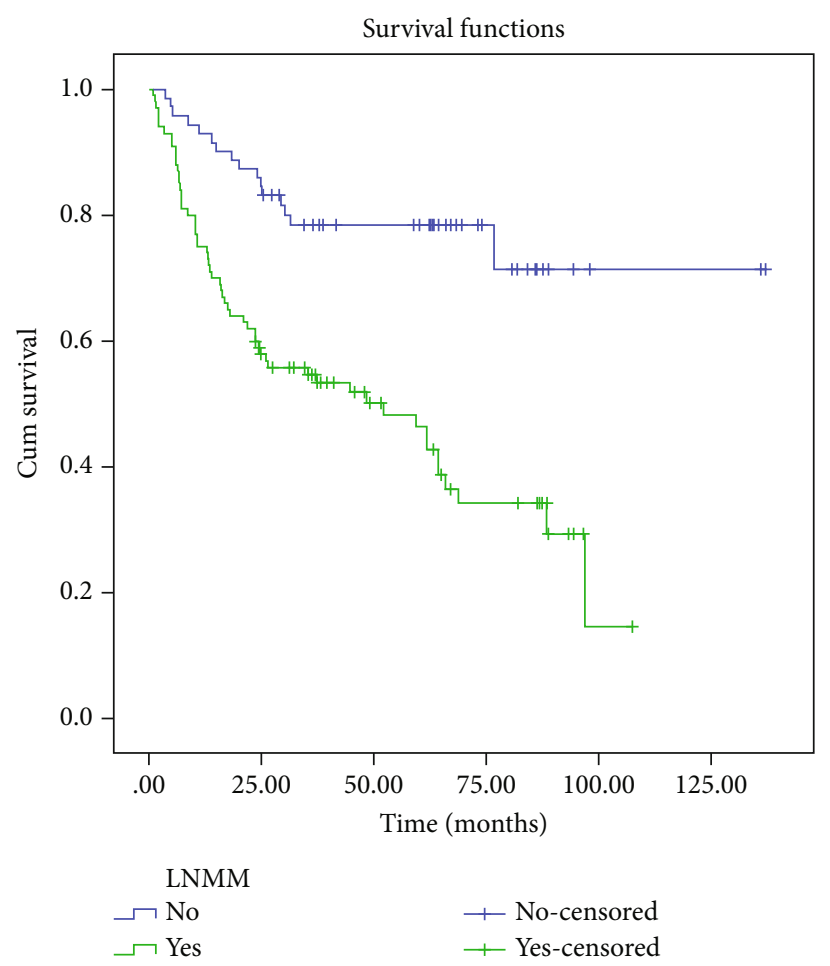

FIgURe 2: Kaplan-Meier disease survival curve according to the presence of LNMM. 5-year overall survival (OS) rate was significantly longer in patients without LNMM vs. with LNMM (76.4\% vs. $42 \%, P<0.001)$.

sis showed that the depth of invasion, TNM staging, lymphatic infiltration, and tumor differentiation were independent risk factors associated with LNMM. Moreover, Cox proportional hazards regression mode demonstrated that LNMM, tumor size, Lauren type, invasion depth, and lymphatic infiltration were independent prognostic factors for long-term survival. Furthermore, our results showed that LNMM had a negative influence on 5-year survival in GC patients $(76.4 \%$ vs. $42 \%, P<0.001)$.

The detection rate of LNMM shows a great difference depending on applied methods and the stage of GC. Maehara et al. showed that the incidence of LNMM ranged from $4.8 \%$ to $36 \%$ in the early-stage GC patients with histologically node-negative [5]. Fukagawa et al. reported 23.5\% of LNMM 
TABle 3: Association of clinicopathological factors with a 5-year survival rate.

\begin{tabular}{|c|c|c|c|}
\hline Clinicopathological factors & No. (\%) & $\begin{array}{l}5 \text {-year survival } \\
\text { rate }(\%)\end{array}$ & $P$ value \\
\hline Gender & & & 0.54 \\
\hline Male & $\begin{array}{c}122 \\
(70.9)\end{array}$ & 60 & \\
\hline Female & $\begin{array}{c}50 \\
(29.1)\end{array}$ & 54.9 & \\
\hline Age & & & 0.95 \\
\hline$\leq 60$ & $\begin{array}{c}98 \\
(57.0)\end{array}$ & 58.1 & \\
\hline$\geq 60$ & $\begin{array}{c}74 \\
(43.0)\end{array}$ & 55.1 & \\
\hline Tumor size (cm) & & & $0.01^{*}$ \\
\hline$\leq 2$ & $\begin{array}{c}31 \\
(18.0)\end{array}$ & 96.8 & \\
\hline $2 \sim 5$ & $\begin{array}{c}79 \\
(45.9)\end{array}$ & 57 & \\
\hline$\geq 5$ & $\begin{array}{c}62 \\
(36.1)\end{array}$ & 35.5 & \\
\hline Tumor differentiation & & & $0.00^{*}$ \\
\hline $\begin{array}{l}\text { High or moderate } \\
\text { differentiation }\end{array}$ & $\begin{array}{c}47 \\
(27.3)\end{array}$ & 87.5 & \\
\hline Low differentiation & $\begin{array}{c}125 \\
(72.7)\end{array}$ & 47 & \\
\hline Level 2 LNMM & & & $0.01^{*}$ \\
\hline Positive & $\begin{array}{c}72 \\
(41.9)\end{array}$ & 42 & \\
\hline Negative & $\begin{array}{c}100 \\
(58.1)\end{array}$ & 76.4 & \\
\hline $\begin{array}{l}\text { The depth of tumor } \\
\text { invasion }\end{array}$ & & & $0.01^{*}$ \\
\hline $\mathrm{T} 1$ & $\begin{array}{c}19 \\
(11.1)\end{array}$ & 86.1 & \\
\hline $\mathrm{T} 2$ & $\begin{array}{c}26 \\
(15.1)\end{array}$ & 72.3 & \\
\hline T3 & $\begin{array}{c}101 \\
(58.7)\end{array}$ & 56.4 & \\
\hline $\mathrm{T} 4$ & $\begin{array}{c}26 \\
(15.1)\end{array}$ & 27.8 & \\
\hline Operation mode & & & $0.07^{*}$ \\
\hline Total gastrectomy & $\begin{array}{c}119 \\
(69.2)\end{array}$ & 59.9 & \\
\hline Distal gastrectomy & $\begin{array}{c}53 \\
(30.8)\end{array}$ & 37.5 & \\
\hline Neural invasion & & & 0.42 \\
\hline Yes & $\begin{array}{c}37 \\
(21.5)\end{array}$ & 51.4 & \\
\hline No & $\begin{array}{c}135 \\
(78.5)\end{array}$ & 57.8 & \\
\hline Lauren classification & & & $0.01^{*}$ \\
\hline Diffusive type & $\begin{array}{c}104 \\
(60.5)\end{array}$ & 48.1 & \\
\hline
\end{tabular}

TABle 3: Continued.

\begin{tabular}{lccc}
\hline Clinicopathological factors & No. (\%) & $\begin{array}{c}\text { 5-year survival } \\
\text { rate (\%) }\end{array}$ & $P$ value \\
\hline Intestinal type & 68 & 69.1 & \\
TNM staging & $(39.5)$ & & $0.01^{*}$ \\
IA & 19 & 86.1 & \\
IB & $(11.1)$ & & \\
IIA & 26 & 72.3 & \\
IIB & $(15.1)$ & & \\
III & 101 & 56.4 & $0.01^{*}$ \\
Lymphatic infiltration & $(58.8)$ & & \\
Yes & 19 & 30.1 & \\
No & $(11.1)$ & 21.8 & \\
& $7(9.9)$ & & \\
\hline
\end{tabular}

LNMM: lymph node micrometastasis; TNM: tumor node metastasis; ${ }^{*} \mathrm{P}<$ 0.05 .

was observed in 34 node-negative GC patients with pT1 tumor by IHC [17]. Yasuda et al. demonstrated that LNMM was detected in 73 nodes (4\%) and 20 patients (31\%) with pT2 and pT3 tumors by IHC [18]. The incidence of LNMM is also closely related to the clinicopathological characteristics of GC patients. The previous study with advanced GC reported that the incidence of MM in lymph nodes increases in proportion to tumor size and the depth of invasion [10]. In our study, the identification rate of LNMM (72/172, 41.9\%) was much higher than the previous studies. Furthermore, we found that with the tumor depth invading, MM occurrence rate corresponding increased $(10.53 \%(2 / 19) \mathrm{T} 1$, $30.77 \%(8 / 26) \mathrm{T} 2,45.54 \%(46 / 101) \mathrm{T} 3$, and $61.54 \%(16 / 26)$ T4). The main reason for explaining this higher result is that advanced stage GC patients occupy the majority of our data. In these patients, the depth of invasion, changes of lymph nodes, and lymphatic invasion may all increase the risk of tumor emboli and thereby increased the risk for LNMM $[19,20]$. Besides the depth of invasion, we also revealed that tumor differentiation, lymphatic infiltration, and TNM staging were independent risk factors for LNMM.

In clinical, lymph node metastasis is considered to be a most reliable prognostic factor that has been widely applied in the TNM staging system to estimate prognosis and guide clinical decision-making. However, some researchers reported that even after undergoing curative surgery and conventional postoperative $\mathrm{pN} 0$ diagnosis does not guarantee against recurrence [21]. Several studies have demonstrated that the presence of LNMM has been identified as an independent risk factor associated with poor outcome in several malignancies including breast [22], colon [23], esophagus [24], gallbladder [25], and lung cancers [26]. Nakajo et al. reported that LNMM was correlated with a significantly lower survival rate in GC patients with $\mathrm{T} 1$ or T2 tumors [27]. 
TABLE 4: Multivariate analysis of the prognostic factors associated with the survival.

\begin{tabular}{|c|c|c|c|c|c|}
\hline Variables & SE & Wald & HR & $95 \% \mathrm{CI}$ & $P$ value \\
\hline Depth of tumor invasion & 0.149 & 5.522 & 1.418 & $1.060-1.897$ & 0.019 \\
\hline Level 2 station LNMM & 0.286 & 10.082 & 2.476 & $1.415-4.334$ & 0.001 \\
\hline Tumor size & 0.230 & 8.779 & 1.978 & $1.260-3.106$ & 0.003 \\
\hline TNM staging & 0.150 & 4.473 & 1.375 & $1.024-1.846$ & 0.034 \\
\hline Lauren classification & 0.472 & 11.017 & 0.209 & $1.083-1.527$ & 0.001 \\
\hline Lymphatic infiltration & 0.272 & 15.219 & 2.893 & $1.697-4.934$ & 0.000 \\
\hline
\end{tabular}

LNMM: lymph node micrometastasis; TNM: tumor node metastasis; HR: hazard ratio; CI: confidence interval; SE: standard error.

Cai et al. found a significant association between LNMM and poor prognosis in patients with T3 GC patients [28]. Although most of studies have shown that LNMM is associated with poor prognosis in GC patients, some researchers still have different findings. Fukagawa et al. showed that the presence of LNMM did not affect survival in a large number of T2 GC patients [17]. In our previous meta-analysis, we performed 18 relevant studies, and the results demonstrated that LNMM had an intimate, significant relationship with a higher risk of disease recurrence and worse survival in GC patients [29]. In the present study, our results indicated that the level 2 station LNMM was indeed associated with poor survival in GC patients. Furthermore, our investigation revealed that LNMM is an independent prognostic indicator for pN0 GC patients with T1-T3. However, it has little prognostic value in T4 patients. The probable reason is that the prognostic significance of LNMM is compromised by peritoneal recurrences which frequently encountered in T4 tumors. Infiltrative tumors have a strong tendency to develop peritoneal seeding when they evolve into T4 tumors. Therefore, it is challenging to confirm the prognostic significance of LNMM in T4 GC patients.

Due to the importance of LNMM in the clinical, how to choose an accurate and sensitive detection tool of LNMM is crucial for disease management and prognosis prediction of GC patients. With the rapid development of molecular biology technology, numerous LNMM detection methods have emerged recently. The IHC staining with AE1/AE3 and TTF-1 is a classic method of detecting LNMM [30]. However, the IHC method was arduous because it requires the remarkable labor of preparing slides and allows senior pathologists to search for tiny tumor cells under the microscope. TRAP-ELISA is a new method with high sensitivity and accuracy for detecting MM. Hu et al. assessed telomerase activity in tumor samples and peritoneal washings from 46 GC patients, and the results showed that telomerase activity was detected in $89.1 \%$ of the GC tissue specimens and $47.8 \%$ of the peritoneal washing sample, which is much higher than those obtained by conventional cytological examinations [14]. In addition, they also revealed that telomerase activity was correlated with tumor grades, depth of tumor invasion, area of serosal invasion, and peritoneal metastasis. This procedure allows a rapid and reproducible analysis of large pools of samples. Although TRAP-ELISA appears to be a useful tool in detecting LNMM, its use in routine examinations is controversial due to the expensive prop- erty. Further research is needed to find the ideal marker with high accuracy and low cost to detect LNMM.

Despite of the satisfactory results, our study have several limitations. First, the present study included only a small sample data from a single institution, multicenter prospective trial that includes a larger number of patients will improve the performance. Second, due to the different proportions of patients with different stages of study, there will be some bias in the final result. Appropriate adjustment of the number of patients in different stages in future studies will reduce this bias. Finally, we only analyzed the MM from the level 2 station lymph nodes, the significance of the level 1 station LNMM is unknown, so all the resected lymph nodes should be investigated in the future.

In conclusion, our results demonstrated that the depth of invasion, TNM staging, lymphatic infiltration, and tumor differentiation were the independent risk factors associated with LNMM. We also revealed that LNMM is a clinically negative prognostic factor in pN0 GC patients. Therefore, for these high-risk patients with LNMM, more sensitive and accurate detection tools may be needed to obtain a precise diagnosis of MM, which is beneficial to develop individualized treatment plans and achieve favorable outcomes.

\section{Data Availability}

The datasets used and analyzed during the current study are available from the corresponding author on reasonable request.

\section{Conflicts of Interest}

The authors declare that there is no conflict of interest.

\section{References}

[1] F. Bray, J. Ferlay, I. Soerjomataram, R. L. Siegel, L. A. Torre, and A. Jemal, "Global cancer statistics 2018: GLOBOCAN estimates of incidence and mortality worldwide for 36 cancers in 185 countries," Ca : a Cancer Journal for Clinicians, vol. 68, no. 6, pp. 394-424, 2018.

[2] P. Karimi, F. Islami, S. Anandasabapathy, N. D. Freedman, and F. Kamangar, "Gastric cancer: descriptive epidemiology, risk factors, screening, and prevention," Cancer Epidemiology, Biomarkers \& Prevention, vol. 23, no. 5, pp. 700-713, 2014.

[3] M. Sasako, T. Sano, S. Yamamoto et al., "D2 lymphadenectomy alone or with para-aortic nodal dissection for gastric 
cancer," The New England Journal of Medicine, vol. 359, no. 5, pp. 453-462, 2008.

[4] K. Ishida, T. Katsuyama, A. Sugiyama, and S. Kawasaki, "Immunohistochemical evaluation of lymph node micrometastases from gastric carcinomas," Cancer, vol. 79, no. 6, pp. 1069-1076, 1997.

[5] Y. Maehara, T. Oshiro, K. Endo et al., "Clinical significance of occult micrometastasis in lymph nodes from patients with early gastric cancer who died of recurrence," Surgery, vol. 119, no. 4, pp. 397-402, 1996.

[6] J. R. Siewert, R. Kestlmeier, R. Busch et al., "Benefits of D2 lymph node dissection for patients with gastric cancer and pN0 and pN1 lymph node metastases," The British Journal of Surgery, vol. 83, no. 8, pp. 1144-1147, 1996.

[7] Y. Zhou, G. J. Zhang, J. Wang, K. Y. Zheng, and W. Fu, "Current status of lymph node micrometastasis in gastric cancer," Oncotarget, vol. 8, no. 31, pp. 51963-51969, 2017.

[8] M. B. Amin, S. B. Edge, F. L. Greene, and D. Byrd, AJCC Cancer Staging Manual, Springer, New York, 8th edition, 2017.

[9] H. Yamamoto, Y. Miyake, S. Noura et al., "Clinical significance of micrometastasis to lymph nodes in gastrointestinal tract cancers," Gan to Kagaku Ryoho, vol. 28, no. 6, pp. 776-783, 2001.

[10] K. Blouhos, K. A. Boulas, and A. Hatzigeorgiadis, "Clinical significance of lymph nodes micrometastasis in conventionally node-negative advanced gastric cancer," Updates in Surgery, vol. 69, no. 3, pp. 425-426, 2017.

[11] H. Sonoda and T. Tani, "Clinical significance of molecular diagnosis for gastric cancer lymph node micrometastasis," World Journal of Gastroenterology, vol. 20, no. 38, pp. 13728-13733, 2014.

[12] R. T. Calado and N. S. Young, "Telomere diseases," The New England Journal of Medicine, vol. 361, no. 24, pp. 2353-2365, 2009.

[13] M. J. Ahn, Y. H. Noh, Y. S. Lee et al., "Telomerase activity and its clinicopathological significance in gastric cancer," European Journal of Cancer, vol. 33, no. 8, pp. 1309-1313, 1997.

[14] X. Hu, H. Wu, S. Zhang, H. Yuan, and L. Cao, "Clinical significance of telomerase activity in gastric carcinoma and peritoneal dissemination," The Journal of International Medical Research, vol. 37, no. 4, pp. 1127-1138, 2009.

[15] Japanese Gastric Cancer Association, "Japanese classification of gastric carcinoma: 3rd English edition," Gastric Cancer, vol. 14, no. 2, pp. 101-112, 2011.

[16] R. González-Quevedo, C. de Juan, M. J. Massa et al., "Detection of telomerase activity in human carcinomas using a trap-ELISA method: correlation with hTR and hTERT expression," International Journal of Oncology, vol. 16, no. 3, pp. 623-628, 2000.

[17] T. Fukagawa, M. Sasako, G. B. Mann et al., "Immunohistochemically detected micrometastases of the lymph nodes in patients with gastric carcinoma," Cancer, vol. 92, no. 4, pp. 753-760, 2001.

[18] K. Yasuda, Y. Adachi, N. Shiraishi, M. Inomata, H. Takeuchi, and S. Kitano, "Prognostic effect of lymph node micrometastasis in patients with histologically node-negative gastric cancer," Annals of Surgical Oncology, vol. 9, no. 8, pp. 771-774, 2002.

[19] T. Arigami, S. Natsugoe, Y. Uenosono et al., "Lymphatic invasion using D2-40 monoclonal antibody and its relationship to lymph node micrometastasis in pN0 gastric cancer," British Journal of Cancer, vol. 93, no. 6, pp. 688-693, 2005.

[20] M. Morita, H. Baba, T. Fukuda et al., "Submucosal gastric cancer with lymph node metastasis," Journal of Surgical Oncology, vol. 68, no. 1, pp. 5-10, 1998.

[21] H. Saito, T. Osaki, D. Murakami et al., "Recurrence in early gastric cancer-presence of micrometastasis in lymph node of node negative early gastric cancer patient with recurrence," Hepato-Gastroenterology, vol. 54, no. 74, pp. 620-624, 2007.

[22] S. E. Clare, S. F. Sener, W. Wilkens, R. Goldschmidt, D. Merkel, and D. J. Winchester, "Prognostic significance of occult lymph node metastases in node-negative breast cancer," Annals of Surgical Oncology, vol. 4, no. 6, pp. 447-451, 1997.

[23] N. N. Rahbari, U. Bork, E. Motschall et al., "Molecular detection of tumor cells in regional lymph nodes is associated with disease recurrence and poor survival in node-negative colorectal cancer: a systematic review and meta-analysis," Journal of Clinical Oncology, vol. 30, no. 1, pp. 60-70, 2012.

[24] S. Natsugoe, J. Mueller, H. J. Stein, M. Feith, H. Höfler, and J. R. Siewert, "Micrometastasis and tumor cell microinvolvement of lymph nodes from esophageal squamous cell carcinoma: frequency, associated tumor characteristics, and impact on prognosis," Cancer, vol. 83, no. 5, pp. 858-866, 1998.

[25] E. Sasaki, M. Nagino, T. Ebata et al., "Immunohistochemically demonstrated lymph node micrometastasis and prognosis in patients with gallbladder carcinoma," Annals of Surgery, vol. 244, no. 1, pp. 99-105, 2006.

[26] J. H. Jeong, N. Y. Kim, and J. S. Pyo, "Prognostic roles of lymph node micrometastasis in non-small cell lung cancer," Pathology, Research and Practice, vol. 214, no. 2, pp. 240-244, 2018.

[27] A. Nakajo, S. Natsugoe, S. Ishigami et al., "Detection and prediction of micrometastasis in the lymph nodes of patients with pN0 gastric cancer," Annals of Surgical Oncology, vol. 8, no. 2, pp. 158-162, 2001.

[28] J. Cai, M. Ikeguchi, M. Maeta, N. Kaibara, and T. Sakatani, "Clinicopathological value of immunohistochemical detection of occult involvement in pT3N0 gastric cancer," Gastric Cancer, vol. 2, no. 2, pp. 95-100, 1999.

[29] Y. Li, P. Du, Y. Zhou et al., "Lymph node micrometastases is a poor prognostic factor for patients in pN0 gastric cancer: a meta-analysis of observational studies," The Journal of Surgical Research, vol. 191, no. 2, pp. 413-422, 2014.

[30] M. Hata, J. Machi, J. Mamou et al., "Entire-volume serial histological examination for detection of micrometastases in lymph nodes of colorectal cancers," Pathology Oncology Research, vol. 17, no. 4, pp. 835-841, 2011. 